\title{
Adherence of obstructive sleep apnoea syndrome patients to positive airway pressure therapy - 10-year follow-up
}

\author{
Samuel Genzora , Jan Praskob, Jakub Vanek ${ }^{\mathrm{b}}$, Amjad Ghazal Asswad', Shayan Nadjarpourd, Milan Sova ${ }^{\mathrm{a}}$
}

\begin{abstract}
Purpose. Continuous positive airway pressure (CPAP) is the most effective therapy for obstructive sleep apnoea syndrome (OSAS). Long-term adherence is necessary; however, it may be widely variable based on current literature, where the predicting factors are also not well defined. The aim of this study was to assess ten-year adherence to CPAP and to define factors influencing it.

Methods. In total, 107 patients (91 males) were enrolled in this study, with an average age of 53.5 years (SD 10.1), with CPAP therapy being indicated (Apnoea-Hypopnoea Index - AHI > 15). Anthropometric and social status data have been obtained as well as the average use of CPAP through device memory.

Results. Sufficient adherence ( $>4 \mathrm{~h}$ per night) was found in $57 \%$ of patients in the first year with no subsequent significant change during the next 9 years. Adherence correlated significantly with the severity of OSAS - AHI $(r=0.321$ over 5 years) and Epworth Sleepiness Scale ( $r=0.317$ over ten years) but did not correlate with the pressure used or the age of the patient. No statistically significant differences have been found between the sexes, the different mask groups, or the social status groups.
\end{abstract}

Conclusion. Good adherence to CPAP therapy in OSAS patients is essential. According to our results, patients with more severe OSAS or more severe sleepiness seem to have better adherence.

Key words: sleep apnoea syndrome, CPAP therapy, adherence to therapy, follow-up, social status

Received: January 14, 2021; Revised: June 15, 2021; Accepted: June 15, 2021; Available online: July 2, 2021

https://doi.org/10.5507/bp.2021.041

(c) 2022 The Authors; https://creativecommons.org/licenses/by/4.0/

${ }^{a}$ Department of Respiratory Medicine, Faculty of Medicine and Dentistry, Palacky University Olomouc and University Hospital Olomouc, Czech Republic

${ }^{b}$ Department of Psychiatry, University Hospital Olomouc and Faculty of Medicine and Dentistry, Palacky University Olomouc, Czech Republic 'Department of Respiratory Medicine West Middlesex University Hospital, London, United Kingdom of Great Britain and Northern Ireland ${ }^{d}$ Faculty of Medicine and Dentistry, Palacky University Olomouc and University Hospital Olomouc, Czech Republic

Corresponding author: Milan Sova, e-mail: milan.sova@email.cz

\section{INTRODUCTION}

Obstructive sleep apnoea syndrome (OSAS) is the most common sleep-related breathing disorder, with a prevalence of almost $10 \%$ (ref. ${ }^{1}$ ) The main nocturnal symptoms are snoring and repetitive breathing cessations (more than 5 times per hour of sleep), and the most critical daytime symptom is excessive sleepiness ${ }^{2}$. Numerous serious comorbidities are associated with this disorder, and it can potentially reduce life expectancy ${ }^{3-5}$. Moreover, OSAS also lowers patients' quality of life ${ }^{6}$.

Of all the possible therapeutic modalities, continuous positive airway pressure (CPAP) therapy still remains the gold standard in OSAS therapy ${ }^{7}$. CPAP therapy leads to an improvement in quality of life $^{8}$, and may reduce certain health risks if the adherence of the patient is sufficient ${ }^{9,10}$. Sufficient adherence is defined by most studies as the use of CPAP for over $4 \mathrm{~h}$ per night on average, however it is unclear if such adherence is enough to achieve the full extent of the possible benefits of the therapy ${ }^{11}$.

There are various factors that could potentially impact CPAP adherence in patients with OSAS including the patients' age, sex, social status, employment status, disease severity and symptoms, the parameters of CPAP therapy, comorbidities, and psychiatric conditions amongst others $^{12-14}$.

This study aims to analyse the adherence of OSAS patients treated with CPAP during long-term follow-up and the potential factors influencing adherence.

\section{Aims of study}

To establish whether CPAP adherence in OSAS therapy will be significantly influenced by:

\section{(a) Objective clinical variables}

AHI (Apnoea-Hypopnoea Index), BMI (Body Mass Index), t90 (percentage time of sleep spent under $90 \%$ of peripheral blood saturation), ODI (Oxygen Desaturation Index)

(b) Demographics

Age, sex, social status, employment status

(c) Subjective evaluation of patient sleepiness measured using the Epworth Sleepiness Scale (ESS)

(d) The pressure (mbar) used during therapy and the type of mask used 


\section{MATERIALS AND METHODS}

This study had a prospective design. The study was approved by the local ethics committee and fulfils the criteria set out by the Declaration of Helsinki.

Patients were enrolled in the period 2005-2008 and were subsequently followed-up over the course of ten years at routine visits.

\section{Inclusion criteria:}

AHI $>15$

Age 18-80

\section{Exclusion criteria:}

Initial refusal of CPAP therapy

Contraindication for CPAP therapy

\section{Course of study:}

Sleep study was performed with respiratory sleep polygraphy (Stardust, Easyscreen)

CPAP titration was performed by autoCPAP machine for 3 nights

Annual follow-up over the course of ten years

Various body features were initially assessed including weight, height, body mass index (BMI) and the circumfer- ence of the neck, waist, and hips. The diagnosis of OSAS was determined using respiratory sleep polygraphy, the recording lasted $7 \mathrm{~h}$ and was evaluated by sleep laboratory technicians according to the current American Academy of Sleep Medicine scoring manual ${ }^{2}$. The optimum therapeutic pressure was titrated using automatic CPAP for at least three nights, during which the patient was hospitalized at the sleep laboratory. The home PAP therapy was initiated only after determining the optimum pressure using the autoCPAP machine during the 3 nights of hospitalization.

The exclusion criterion was the presence of a contraindication for CPAP therapy (CPAP intolerance, cerebrospinal fluid leak, chronic sinusitis, etc.). Good adherence to the therapy was defined as more than four hours of CPAP use per night.

The ESS was used to assess the sleepiness of patients with OSAS (ref. ${ }^{15}$ ).

Patients were further divided into sub-groups according to their social status - unemployed, retired, manual workers, clerks, patients with a university degree, and professional drivers.

IBM SPSS Statistics software version 25.0 was used for data analysis. The Pearson correlation index evaluated

Table 1. Study group characteristics.

\begin{tabular}{llccc}
\hline & Average & Median & Minimum & Maximum \\
\hline Age (years) & $53.5 \pm 10.1$ & 55.0 & 21.0 & 73.0 \\
BMI $\left(\mathrm{kg} / \mathrm{m}^{2}\right)$ & $36.7 \pm 6.4$ & 35.8 & 26.0 & 60.7 \\
ESS (points) & $10.6 \pm 5$ & 10.0 & 3.0 & 24.0 \\
AHI (per hour) & $54.6 \pm 23$ & 53.4 & 15.1 & 119.1 \\
t90 (\%) & $36.3 \pm 23.7$ & 29.2 & 1.04 & 99 \\
PAP (mbars) & $8.3 \pm 2$ & 8.5 & 4 & 15.5 \\
PAP therapy use (hours) & $4.97 \pm 2.3$ & 5.33 & 0 & 12.97 \\
\hline
\end{tabular}

Age, age of patients at the beginning of follow-up; BMI, Body Mass Index; ESS, Epworth Sleepiness Scale; AHI, Apnoea-Hypopnoea Index; t90, \% of total sleep time spent under $90 \%$ blood saturation; PAP, pressure used during positive airway pressure therapy (mbar); PAP therapy use, average number of hours of PAP therapy per night.

Table 2. CPAP adherence in different social groups - employed, retired, and retired due to disability.

\begin{tabular}{|c|c|c|c|c|c|}
\hline \multirow{2}{*}{\multicolumn{2}{|c|}{$\begin{array}{l}\text { Average CPAP use per } \\
\text { night, at year of follow-up }\end{array}$}} & \multicolumn{3}{|c|}{ Social group/Employment status } & \multirow[b]{2}{*}{$P$} \\
\hline & & $\begin{array}{l}\text { Employed } \\
\quad \mathrm{n}=72\end{array}$ & $\begin{array}{c}\text { Retired } \\
\mathrm{n}=25\end{array}$ & $\begin{array}{l}\text { Retired due to disability } \\
\qquad \mathrm{n}=10\end{array}$ & \\
\hline \multirow[t]{3}{*}{1 Year } & Median & 4.42 & 4.99 & 4.86 & \multirow{3}{*}{0.634} \\
\hline & Minimum & 0.11 & 0.58 & 0.07 & \\
\hline & Maximum & 9.39 & 11.14 & 8.57 & \\
\hline \multirow{3}{*}{3 Years } & Median & 5.01 & 5.68 & 6.19 & \multirow{3}{*}{0.566} \\
\hline & Minimum & 0.19 & 0.39 & 1.10 & \\
\hline & Maximum & 12.97 & 8.01 & 9.80 & \\
\hline \multirow[t]{3}{*}{5 Years } & Median & 5.56 & 5.70 & 6.73 & \multirow{3}{*}{0.742} \\
\hline & Minimum & 0.75 & 1.83 & 1.39 & \\
\hline & Maximum & 10.74 & 8.06 & 10.06 & \\
\hline \multirow[t]{3}{*}{10 Years } & Median & 5.29 & 5.55 & 4.77 & \multirow{3}{*}{0.798} \\
\hline & Minimum & 0.36 & 1.55 & 1.47 & \\
\hline & Maximum & 9.73 & 8.06 & 10.80 & \\
\hline
\end{tabular}


Table 3. CPAP adherence in different social groups - differences between groups in the average length of CPAP use per night.

\begin{tabular}{|c|c|c|c|c|c|c|c|}
\hline \multirow{2}{*}{\multicolumn{2}{|c|}{$\begin{array}{l}\text { Average CPAP use per night, } \\
\text { at year of follow-up }\end{array}$}} & \multicolumn{5}{|c|}{ Social group/Employment status } & \multirow[b]{2}{*}{$P$} \\
\hline & & \multirow{2}{*}{$\begin{array}{c}1 \\
\mathrm{n}=35 \\
3.23\end{array}$} & \multirow{2}{*}{$\begin{array}{c}2 \\
n=59 \\
4.90\end{array}$} & \multirow{2}{*}{$\begin{array}{c}3 \\
n=15 \\
4.33\end{array}$} & \multirow{2}{*}{$\begin{array}{c}\begin{array}{c}4 \\
n=19\end{array} \\
5.29\end{array}$} & \multirow{2}{*}{$\begin{array}{c}5 \\
\mathrm{n}=9 \\
2.59\end{array}$} & \\
\hline 1 Year & Median & & & & & & \multirow{3}{*}{0.509} \\
\hline & Minimum & 3.23 & 0.07 & 0.74 & 0.63 & 0.11 & \\
\hline & Maximum & 3.23 & 11.14 & 9.39 & 7.82 & 6.45 & \\
\hline \multirow[t]{3}{*}{3 Years } & Median & \multirow{3}{*}{ No data } & 5.70 & 5.43 & 5.54 & 3.19 & \multirow{3}{*}{0.564} \\
\hline & Minimum & & 0.39 & 1.00 & 0.19 & 0.86 & \\
\hline & Maximum & & 12.97 & 8.92 & 7.04 & 7.23 & \\
\hline \multirow[t]{3}{*}{5 Years } & Median & \multirow{3}{*}{ No data } & 5.87 & 4.92 & 5.76 & 3.70 & \multirow{3}{*}{0.781} \\
\hline & Minimum & & 0.75 & 1.91 & 2.10 & 1.94 & \\
\hline & Maximum & & 10.74 & 8.93 & 8.29 & 9.34 & \\
\hline \multirow[t]{3}{*}{10 Years } & Median & \multirow{3}{*}{ No data } & 5.37 & 4.89 & 5.52 & 4.15 & \multirow{3}{*}{0.734} \\
\hline & Minimum & & 1.55 & 1.71 & 0.36 & 2.24 & \\
\hline & Maximum & & 10.80 & 9.73 & 8.28 & 7.71 & \\
\hline
\end{tabular}

1, unemployed; 2, manual worker; 3 , clerk; 4, patients with a university degree; 5 , professional driver.

the correlation of quantitative parameters with adherence. Kruskal-Wallis test or Mann-Whitney U-test evaluated the differences in adherence across various groups. To evaluate the statistical significance of the change in adherence, the McNemar test was used. Statistical significance was evaluated at the level 0.05 in all methods. Binary logistic regression was used to predict adherence after ten years of CPAP treatment.

\section{RESULTS}

The total number of subjects involved in this study was 107 of which 91 were males ( $85 \%)$, and 16 females (15\%). Study group characteristics are summarized in Table 1.

More than $4 \mathrm{~h}$ of average CPAP use per night was achieved in $57 \%$ of patients after 1 year, $57.5 \%$ after 3 years, $58.6 \%$ after 5 years, and $57.3 \%$ after ten years of CPAP therapy. No significant changes in CPAP adherence were found using the McNemar test when comparing first-year follow-up adherence with adherence at subsequent check-ups.

During the follow-up period, 13 patients died with an average age of 64.2 (49-74) and an average CPAP therapy length of 5.15 years (1-9 years). A further 21 patients were withdrawn completely from therapy or had no adherence (mostly in the first year of follow-up).

Neither the patients' age nor BMI was found to correlate with CPAP adherence. ESS had a weak, but statistically significant correlation with CPAP adherence both after 1 year, and ten years and was close to statistical significance at other intervals. The AHI correlated significantly with the rate of adherence to CPAP at 1,3 , and 5 years of follow-up, but significance was not proven at ten years of follow-up. The correlation coefficient showed weak to moderate correlation. Similarly, ODI correlated with CPAP adherence at 3 and 5 years of follow-up, but not after 1 year and ten years. T90 had a significant, though weak correlation with CPAP adherence only at ten years of follow-up. The pressure used during therapy did not correlate with adherence at any interval.

No significant differences in the average amount of time of CPAP use per night were found when comparing the groups with different mask types (nasal versus oronasal mask: $\mathrm{p}$-values using the Mann-Whitney t-test for CPAP therapy adherence at set study intervals are as follows; 1 year $-0.92 ; 3$ years $-0.74 ; 5$ years -0.152 ; ten years -0.293 ).

There were no statistically significant differences in adherence between employed patients (66) and retired patients (pensioner - 25 patients, disability pension 9 patients) (Table 2).

There was no significant difference found in the average length of CPAP use per night in different social groups (unemployed - 2 patients, manual workers - 59, clerks -15 , patients with a university degree -19 , professional driver - 9) (Table 3).

\section{Regression analyses \\ Average daily hours on CPAP treatment}

To detect the most significant variables associated with ten years of adherence measured by average daily hours on CPAP treatment, a multiple regression analysis (backward stepwise regression) was carried out. The daily average number of hours on CPAP treatment was taken as the dependant variable. The independent variables were the patient's age, ESS, AHI, ODI, t90, average night oxygen saturation, BMI, and pressure of CPAP used. During nine steps of the backward stepwise regression four factors endured, namely, ESS, AHI, ODI, and t90, which statistically had a significant correlation to the average daily hours on PAP treatment. 


\section{Adherence versus non-adherence after ten years of CPAP treatment}

A binary logistic regression (method: Backward Stepwise - Wald) was used to predict adherence after ten years of CPAP treatment. The variables entered at step 1 were age, ESS, AHI, ODI, t90, average night oxygen saturation and the presence of a psychiatric comorbidity. Nagelkerke's R-square was calculated as 0.194 .

The next stage of the logistic regression showed that after nine steps only ESS, AHI and ODI had a statistically significant impact on ten-year adherence. No other evaluated regressors were detected after nine steps (table 4).

\section{DISCUSSION}

Our investigation aimed to examine the adherence of patients with OSAS treated with CPAP during ten years of follow-up and to determine the clinical factors influencing adherence.

Tan et al. carried out a prospective study of 2160 patients which revealed that patients with mild to moderate OSAS tend to opt for surgery or adjunctive treatments, with $38.4 \%$ rejecting all forms of treatment ${ }^{14}$. From those who chose CPAP therapy (34.8\% of all patients, i.e., 751 patients) only $50.7 \%$ (381) kept a good adherence to the therapy after 1 month. In the follow-up of this group, $78.5 \%$ (299) were adherent after 1 year - this is only $13.8 \%$ of all OSAS patients included in the study. Age, BMI, and normal ESS were predictors of poor adherence; in our study, there was no significant correlation found between age and adherence to the treatment. Compared to the study performed by Tan et al. this study found a better adherence rate in the population followed $(43 \%$ in the Tan et al. study compared to $57.3 \%$ found by this study after 1 year). This study, in contrast to the study by Tan et al. did not find higher age to be a predictor of poor adherence and neither study found any differences in adherence between the sexes.

Gagnadoux et al. followed-up a total of 1104 patients for an average period of 504 days, finding adherence to CPAP therapy to be at $59 \%$ (ref. ${ }^{13}$ ). They identified four factors that influenced CPAP adherence - AHI, BMI, employment status, and marital status. However, age, gender, ESS score, depressive syndrome, associated cardiovascular comorbidities, educational attainment, and occupation category were not found to influence CPAP adherence. In the study by Gagnadoux et al. neither age nor gender were deemed as predictors of CPAP adherence which agrees with the findings of our study. Similarly, the rates of adherence with CPAP were comparable in both studies.

In the study by Gagnadoux et al. the educational attainment and occupation category did not influence CPAP adherence. This is in contrast to the findings of Campbell et al. whose study showed the major impact of social status on CPAP adherence in OSAS patients in New Zealand (214 patients in total) (ref. ${ }^{12}$ ). That study found that patients with poor socioeconomic status and those not of European origin had significantly lower treatment adherence (average follow-up: 504 \pm 251 days).
Table 4. Logistic regression - prediction of the adherence after ten years of CPAP treatment (variables not in the Equation).

Step 0

\begin{tabular}{lccc}
\hline Variable & Score & $\begin{array}{c}\text { Degrees of } \\
\text { freedom }\end{array}$ & $P$ \\
\hline Age (years) & 1.676 & 1 & 0.195 \\
ESS (points) & 6.234 & 1 & 0.013 \\
AHI (per hour) & 4.423 & 1 & 0.035 \\
ODI & 6.603 & 1 & 0.010 \\
t90 (\%) & 2.609 & 1 & 0.106 \\
Average oxygen saturation (\%) & 2.271 & 1 & 0.132 \\
BMI (kg/m $\left.{ }^{2}\right)$ & 0.771 & 1 & 0.380 \\
CPAP (pressure in mbar) & 1.026 & 1 & 0.311 \\
Overall Statistics & & 8 & \\
\hline
\end{tabular}

Adherence to CPAP treatment can be influenced by many factors, particularly by the psychological characteristics of the patient, such as hope, anxiety, mood, or personality traits. These factors have already been studied in connection with OSAS (ref. ${ }^{16}$ ) but not regarding CPAP adherence. Other studies that would assess the effect of these factors on treatment adherence could help in the search for strategies to increase adherence to the treatment.

Inoue et al. found in a study of 711 Japanese patients with an AHI over 20 that 543 (76.3\%) of them started CPAP treatment ${ }^{17} .484$ patients continued to use CPAP after 2 months $(89.1 \%$ of those who started CPAP treatment) and 219 remained compliant (more than 4 hours of CPAP use per night on average) at 1 year (30.8\%). Patients with good adherence after 1 year had a higher AHI and BMI, though no differences were found regarding the sex or age of the patients.

According to our results, no significant differences between different social status groups were found in our correlation analyses. This could possibly be because the patients in the study groups were not so dissimilar regarding their social status.

The parameters of OSAS severity (AHI, t90 and ODI) were shown to have weak, but statistically significant correlations with CPAP adherence in our study.

When looking for the most critical variables linked to ten years of adherence measured by average daily hours of CPAP treatment using multiple regression analyses, two factors appeared to be significant, namely ESS and $\mathrm{t} 90$.

When dividing patients into two groups, according to adherence (more than and less than 4 hours use per night on average), the statistically significant predictors of ten-year adherence were ESS and ODI. This agrees with the Tan et al. study where normal ESS was a predictor of poor adherence ${ }^{14}$. In our study, a higher BMI was not associated with better adherence, Gagnadoux et al. identified both AHI and BMI as positive factors in CPAP adherence $^{13}$.

Similarly, Inoue et al. found no impact of sex and age on CPAP adherence ${ }^{17}$, which is consistent with the results of our study. Both the AHI and BMI correlated signifi- 
cantly with CPAP adherence at 1 year of follow-up. Good adherence (more than 4 hours use per night) was found in $45.2 \%$ of patients, which is lower than in our study.

It was proposed that daily experiences of subjective negative feelings of fatigue, sleepiness and lack of concentration as measured by the ESS would motivate the patient to use CPAP regularly. This hypothesis was confirmed at 1 year and ten years of follow-up. Similarly, Tan et al. had a similar finding where normal ESS was a predictor of poor CPAP adherence ${ }^{14}$. In contrast, Gagnadoux et al. ${ }^{13}$ and Campbell et al. ${ }^{12}$ found no correlation between ESS and CPAP adherence.

The pressure of CPAP and the type of mask used do not influence adherence to the therapy, which was confirmed at all points of follow-up. This was consistent with the findings of Schirlaw et al. ${ }^{18}$ and Sova et al. ${ }^{19}$. On the other hand, Rowland et al. revealed a significantly higher quality of sleep in the nasal mask group, though no significant differences in CPAP adherence between the nasal and oronasal mask groups were found ${ }^{20}$.

In contrast, Borrel et al. found a higher adherence in the nasal mask group in the 2311 patients studied and thus recommends the use of this mask type as a first choice ${ }^{21}$. In our study, we found no significant differences in adherence with CPAP therapy between the nasal and oronasal mask types, however this could be due to the small size of the subgroup using the oronasal mask (18 patients).

The pressure used in the CPAP therapy did not influence CPAP adherence according to Weaver et al. (ref. ${ }^{22}$ ), which is consistent with the findings of our study.

The retrospective observational study of Dzierzewski et al. revealed that the most important factor for CPAP adherence is self-efficacy beliefs of the patients in the therapy ${ }^{23}$. Patients with a profound trust in CPAP therapy (assessed with a questionnaire) showed better adherence when using higher positive pressures in therapy, whereas in the rest of the patients, no correlations between the pressures of CPAP used and adherence were found. Weaver et al. similarly revealed no influence of the pressure used in CPAP therapy on adherence ${ }^{22}$. The authors concluded that a possible way to improve adherence to the therapy is the use of behavioural interventions like cognitive behavioural therapy.

In post-hoc analysis of the SAVE (Sleep Apnoea Cardiovascular Endpoints) study, Van Ryswyk et al. ${ }^{24}$ found in a group of 1121 participants over 24 months of follow-up, that the most important predictors of adherence to CPAP therapy were the adherence rate in the first month of follow-up, the ESS at the beginning of therapy, and the objective parameters of OSAS severity (AHI, ODI, t90). This is in concordance with our results, as ESS, ODI, AHI, and t90 all correlated with CPAP adherence. Similarly, adherence to therapy in our study did not change during our follow-up which is consistent with the results of Van Ryswyk et al. ${ }^{24}$.

Comparing long-term CPAP adherence in OSA patients to the adherence to the treatment in other diseases is an interesting issue to discuss. Sanduzzi et al. studied long-term adherence to the pharmacotherapy in chron- ic obstructive pulmonary disease (COPD) - the major conclusions of the meta-analysis were that the lack of perceived benefit led to $30 \%$ of patients with COPD to discontinue their therapy ${ }^{25}$. Other important factors in COPD adherence were the frequency and simplicity of the therapy administration. This was in concordance with Bozek et al. who found in a population of elderly asthmatic patients that only $21 \%$ had a good adherence ${ }^{26}$. Proper monitoring of asthma by the use of a diary, together with estimation of depression symptoms and cognition status could increase the adherence and control of asthma in this group of patients. Similarly, Ni et al. studied in their systematic review long-term adherence to cardioprotective pharmacotherapy in secondary prevention of coronary heart disease ${ }^{27}$. The drug adherence was considerably low in numerous involved studies, e.g. in the case of betablockers only $49 \%$ of patients consistently used them following discharge and $22 \%$ of them did not commence the use of them at all. The most important tool to improve the adherence of patients is their education.

This study has several limitations. All patients were examined by respiratory polygraphy, without performing complete polysomnography with EEG. There might be a possible bias in the overestimation or underestimation of OSA severity in some patients. On the other hand, this method is widely used and is considered a suitable diagnostic method for OSA. Another possible limitation may be the use of automatic CPAP titration instead of manual CPAP titration with polysomnography. According to the meta-analysis of Gao et al. automatic titration was as effective as standard manual titration in improving AHI and somnolence of the patients ${ }^{28}$. Therefore, we expect that the automatic CPAP titration does not have an impact on CPAP adherence in OSA patients.

This study also only has a relatively small number of patients, especially when considering the different patient groups. All the remaining patients will be followed-up further and the study will continue at our department as a prospective observational study.

\section{CONCLUSION}

According to our results, it seems that patients with more severe OSAS or more serious symptoms have better adherence. Other possible factors seem to have no significant influence on adherence to the therapy. Factors that might influence adherence to the therapy need to be further studied and evaluated. Specific management of different groups of patients might improve their CPAP adherence.

\section{ABBREVIATIONS}

AHI, Apnoea-Hypopnoea Index; BMI, Body Mass Index; COPD, Chronic Obstructive Pulmonary Disease; CPAP, Continuous Positive Airway Pressure; ESS, Epworth Sleepiness Scale; ODI, Oxygen Desaturation 
Index; OSAS, Obstructive Sleep Apnoea Syndrome; SAVE, Sleep Apnoea Cardiovascular Endpoints.

Acknowledgement: Supported by Ministry of Health, Czech Republic - conceptual development of research organization (FNOL, 00098892). Authors would like to thank Dr. Jana Zapletalova for statistical analysis.

Author contributions: GS, SM, PJ, VJ: designed the study and participated on data collection, analysis, and interpretation as well as on the preparation of the manuscript; GAA, SN: participated on data analysis, manuscript preparation, and language correction.

Conflicts of interest statement: Authors declare no conflict of interest regarding this manuscript

Ethics approval: The study was approved by Palacky University, Czech Republic, Ethics Committee (76/08).

\section{REFERENCES}

1. Ferini-Strambi L, Fantini ML, Castronovo C. Epidemiology of obstructive sleep apnea syndrome. Minerva Med 2004;95(3):187-202.

2. American Academy of Sleep Medicine. International classification of sleep disorders, 3rd diagnostic and coding manual. Westchester: American Academy of Sleep Medicine. 2014

3. Abumuamar AM, Newman D, Dorian P, Shapiro CM. Cardiac effects of CPAP treatment in patients with obstructive sleep apnea and atrial fibrillation. J Interv Card Electrophysiol 2019;54(3):289-97.

4. Barcelo A, Pierola J, Lopez-Escribano $H$, de la Pena M, Soriano JB, Alonso-Fernandez A, Ladaria A, Agusti A. Telomere shortening in sleep apnea syndrome. Respir Med 2010;104(8):1225-9.

5. Catalan-Serra P, Campos-Rodriguez F, Reyes-Nunez N, SelmaFerrer MJ, Navarro-Soriano C, Ballester-Canelles M, Soler-Cataluna $\mathrm{JJ}$, Roman-Sanchez P, Almeida-Gonzalez CV, Martinez-Garcia MA. Increased Incidence of Stroke, but Not Coronary Heart Disease, in Elderly Patients With Sleep Apnea. Stroke 2019;50(2):491-4.

6. Andrade L, Paiva T. Ambulatory Versus Laboratory Polysomnography in Obstructive Sleep Apnea: Comparative Assessment of Quality, Clinical Efficacy, Treatment Compliance, and Quality of Life. J Clin Sleep Med 2018;14(8):1323-31.

7. Young D, Collop N. Advances in the treatment of obstructive sleep apnea. Curr Treat Options Neurol 2014;16(8):305.

8. Serrano Merino J, Perula de Torres LA, Bardwell WA, Munoz Gomez R, Roldan Villalobos A, Feu Collado N, Ruiz-Moral R, Jurado-Gamez B. Impact of Positive Pressure Treatment of the Airway on HealthRelated Quality of Life in Elderly Patients With Obstructive Sleep Apnea. Biol Res Nurs 2018;20(4):452-61.

9. Caples SM, Mansukhani MP, Friedman PA, Somers VK. The impact of continuous positive airway pressure treatment on the recurrence of atrial fibrillation post cardioversion: A randomized controlled trial. Int J Cardiol 2019;278:133-6.

10. Peker Y, Balcan B. Cardiovascular outcomes of continuous positive airway pressure therapy for obstructive sleep apnea. J Thorac Dis 2018;10(Suppl 34):S4262-S79.

11. McEvoy RD, Antic NA, Heeley E, Luo Y, Ou Q, Zhang X, Mediano O, Chen R, Drager LF, Liu Z, Chen G, Du B, McArdle N, Mukherjee S, Tripathi M, Billot L, Li Q, Lorenzi-Filho G, Barbe F, Redline S, Wang J, Arima H, Neal B, White DP, Grunstein RR, Zhong N, Anderson CS. CPAP for Prevention of Cardiovascular Events in Obstructive Sleep Apnea. N Engl J Med 2016;375(10):919-31.
12. Campbell A, Neill A, Lory R. Ethnicity and socioeconomic status predict initial continuous positive airway pressure compliance in New Zealand adults with obstructive sleep apnoea. Intern Med J 2012;42(6):e95-101.

13. Gagnadoux F, Le Vaillant M, Goupil F, Pigeanne T, Chollet S, Masson $\mathrm{P}$, Humeau MP, Bizieux-Thaminy A, Meslier N. Influence of marital status and employment status on long-term adherence with continuous positive airway pressure in sleep apnea patients. PloS One 2011;6(8):e22503.

14. Tan B, Tan A, Chan YH, Mok Y, Wong HS, Hsu PP. Adherence to Continuous Positive Airway Pressure therapy in Singaporean patients with Obstructive Sleep Apnea. Am J Otolaryngol 2018;39(5):501-6.

15. Johns MW. Sleepiness in different situations measured by the Epworth Sleepiness Scale. Sleep 1994;17(8):703-10.

16. Hobzova M, Hubackova L, Vanek J, Genzor S, Ociskova M, Grambal A, Prasko J. Cognitive function and depressivity before and after cpap treatment in obstructive sleep apnea patients. Neuro Endocrinol Lett 2017;38(3):145-53.

17. Inoue A, Chiba S, Matsuura K, Osafune H, Capasso R, Wada K. Nasal function and CPAP compliance. Auris Nasus Larynx 2019;46(4):54858.

18. Shirlaw T, Duce B, Milosavljevic J, Hanssen K, Hukins C. A randomised crossover trial comparing nasal masks with oronasal masks: No differences in therapeutic pressures or residual apnea-hypopnea indices. J Sleep Res 2018:e12760.

19. Sova M, Valisova Z, Hobzova M. Adherence to continuous positive airway pressure therapy of obstructive sleep apnea syndrome - An observational study. Stud Pneumol Phthiseol 2016;76(6):219-25.

20. Rowland S, Aiyappan V, Hennessy C, Catcheside P, Chai-Coezter CL, McEvoy RD, Antic NA. Comparing the Efficacy, Mask Leak, Patient Adherence, and Patient Preference of Three Different CPAP Interfaces to Treat Moderate-Severe Obstructive Sleep Apnea. J Clin Sleep Med 2018;14(1):101-8.

21. Borel JC, Tamisier R, Dias-Domingos S, Sapene M, Martin F, Stach B, Grillet Y, Muir JF, Levy P, Series F, Pepin JL. Type of mask may impact on continuous positive airway pressure adherence in apneic patients. PloS One 2013;8(5):e64382.

22. Weaver TE, Grunstein RR. Adherence to continuous positive airway pressure therapy: the challenge to effective treatment. Proc Am Thorax Soc 2008;5(2):173-8.

23. Dzierzewski JM, Wallace DM, Wohlgemuth WK. Adherence to Continuous Positive Airway Pressure in Existing Users: Self-Efficacy Enhances the Association between Continuous Positive Airway Pressure and Adherence. J Clin Sleep Med 2016;12(2):169-76.

24. Van Ryswyk E, Anderson CS, Antic NA, Barbe F, Bittencourt L, Freed R, Heeley E, Liu Z, Loffler KA, Lorenzi-Filho G, Luo Y, Margalef MJM, McEvoy RD, Mediano O, Mukherjee S, Ou Q, Woodman R, Zhang X, Chai-Coetzer CL. Predictors of long-term adherence to continuous positive airway pressure in patients with obstructive sleep apnea and cardiovascular disease. Sleep 2019;42(10):152.

25. Sanduzzi A, Balbo P, Candoli P, Catapano GA, Contini P, Mattei A, Puglisi G, Santoiemma L, Stanziola AA. COPD: adherence to therapy. Multidiscip Respir Med 2014;9(1):60.

26. Bozek A, Jarzab J. Adherence to asthma therapy in elderly patients. J Asthma 2010;47(2):162-5.

27. Ni Z, Dardas L, Wu B, Shaw R. Cardioprotective medication adherence among patients with coronary heart disease in China: a systematic review. Heart Asia 2019;11(2):e011173.

28. Gao W, Jin Y, Wang Y, Sun M, Chen B, Zhou N, Deng Y. Is automatic CPAP titration as effective as manual CPAP titration in OSAHS patients? A meta-analysis. Sleep Breath 2012;16(2):329-40. 